\title{
O CAPITAL SOCIAL NO DESENVOLVEMENTO LOCAL COMUNITARIO. UN ESTUDO EN COMUNIDADES RURAIS DE BOLIVIA
}

Dante Ayaviri Nina

,dayaviri@unach.edu.ec.

Gabith Miriam Quispe Fernández

gquispe@unach.edu.ec.

María Eugenia Borja Lombeida

mborja@unach.edu.ec

Facultade de Ciencias Políticas e Administrativas,

Universidade Nacional de Chimborazo, Bolivia

\section{RESUMO}

A investigación estuda as dimensións do capital social no contexto das comunidades rurais e o seu desenvolvemento local, dadas as potencialidades e recursos existentes nos territorios. A metodoloxía aplicada é a partir da revisión teórica interpretativa das diversas formulacións e as dimensións do capital social neste eido. O estudo identifica ás redes como un elemento destacable no substrato do tecido asociativo para o desenvolvemento local, as organizacións comunitarias, como formas de ralacionamento fortalecidas como principio de xestión colectiva e cooperación, e, por último, a confianza cara ás organizacións nas súas actuacións e intervencións nos procesos de desenvolvemento comunitario, onde os actores locais xogan un papel relevante nos procesos de desenvolvemento local das comunidades rurais. Así, o estudo conclúe facendo especial énfase nas dimensións como mecanismos e elementos que determinan 0 desenvolvemento local baseados na construción de relacións no capital social e o desenvolvemento local comunitario.

Palabras chave: Capital social, dimensións, desenvolvemento local, comunidades rurais.

\section{The Social Capital in local community development. A study in rural communities in Bolivia}

\section{ABSTRACT}

The research studies the dimensions of social capital in the context of rural communities and their local development, given the potential and resources in the territories. The applied methodology is based on the theoretical revision of the different approaches and the dimensions of social capital in this area. The study identifies networks as a prominent element in the substrate of the associative fabric for local development, community organizations, with strengthened relationships as a principle of collective management and cooperation, and finally, the trust in organizations, in their actions and interventions in community development processes, where local actors play a relevant role in the local development processes of rural communities. Thus, the study concludes with a special emphasis on dimensions as mechanisms and elements that determine local development based on building relationships in social capital and local community development.

Key words: Social capital, dimensions, local development, rural communities.

\section{INTRODUCIÓN}

O capital social é o conxunto de valores ou normas que comparten os membros dun colectivo baseados na cooperación (Lesser, 2000; Fukuyama, 2001); é dicir, estes grupos procuran un traballo conxunto en favor dos integrantes, suman esforzos e sinerxías cuxa característica é a comprensión, honestidade, tolerancia e reciprocidade como as forzas axiolóxicas do capital social (Campbell e Jovchelovitch, 2000); polo tanto, a capacidade 
asociativa conduce a unha práctica dos valores e principios de colaboración (Gutiérrez, 2016). Matus (1986) e Falleto (1995) afirman que se debe crear a necesidade de construír a viabilidade colaborativa e organizacional que contribúan no desenvolvemento, outros, en troques, afirman que dende as redes, confórmase o capital social, elemento relevante no desenvolvemento social (Schiff, 1992; Stone, 2000; Winter, 2000; Delhey e Newton, 2002; Sobel, 2002; Roche, 2004; Van Deth, 2008) e económico das rexións (Mavares et al., 1999; Ibañez, 2004; Martinez et al., 2010; Sánchez, 2013) que, á súa vez, permite a construción de sociedades con liberdades (PNUD, 1998; Román et al., 2013). Como consecuencia, o capital social enmárcase no eido do desenvolvemento e os seus compoñentes que acompañan os procesos de transformación das sociedades e o seu crecemento (Scull, 200; Sudarsky, 2004; Kingston e Caballero, 2005), e consecuentemente, revela as actuacións dos actores e axentes do desenvolvemento a través da participación e implicación dos individuos e as redes nos procesos (Falleto e Martner, 1990; Putnam, 2001; Haz, 2015).

Haz (2015: p 30) sinala que "a primeira teorización sobre o capital social outorgouse a James Coleman (1988) e Glenn Loury (1977), que empregaron este concepto para referirse aos recursos que están vencellados ás relacións familiares. Porén, o desenvolvemento teórico en profundidade do capital social corresponde ás obras de Pierre Bourdieu (1980; 1988), James Coleman (1988; 2011) e Robert Putnam (1993; 2002; 2003)". Porén, o estudo do capital social pode ser abordado dende dous enfoques, o estruturalista e comunitario ou culturalista (Coleman, 2011; Haz, 2015). O primeiro considerado como un conxunto de bens dispoñibles para a aplicación en redes (Bourdieu, 1988; Coleman, 2011); a segunda, corresponde á escola comunitaria que procura unha relación entre o desenvolvemento comunitario, sociedade civil e capital social (Putnam, 2001), en ámbolos dous casos, aínda existen baleiros no contido e existen espazos para seguir investigando na temática (Levi, 1996; Kliksber, 2000; Portes, 2000; Lin, 2001; Pena e Sánchez, 2013).

No eido do desenvolvemento comunitario, foi Putnam (2001) quen establece unha relación entre o capital social e o eido local comunitario, e formula unha visión sociocomunitaria orientada ás organizacións comunitarias, que son organizacións locais propias dunha comunidade cuxo propósito é promover os obxectivos económicos ou sociais da poboación (Carpio, 2001; Gutiérrez, 2016). Así, no estudo do capital social interveñen factores e elementos que configuran unha sociedad e o seu contexto (Posner e Boix, 2000; Moyano, 2002; Putnam, 2003; Ibañez, 2004; Van Deth, 2008; Urteaga, 2013; Dodd et al., 2015). Tamén o capital social é abordado dende o eido das redes (Guía, 1999; Margarida e Rego, 2003; Moreno, 2007; Martí e Lozares, 2008; Gutiérrez e Flores, 2011; Ballester et al., 2014), as organizacións (Guinalíu, 2003; Forni et al., 2012, 2013; Mendieta e Araujo, 2012) e a confianza (Thiébault, 2003; Pena e Sánchez, 2005; Gordon, 2005; Frías et al., 2011; Mendieta e Araujo, 2012; Martínez-Cárdenas et al., 2015), estas dimensións aplicadas no contexto rural deberían contribuír nos procesos de desenvolvemento local, obxectivo que buscan as comunidades rurais e o conxunto da sociedade.

Neste contexto, o capital social incide na cohesión comunitaria baseada nas redes de organizacións locais comunitarias, participación dos actores, solidariedade e pertenza (Calloway e Nadlicki, 2000; Muntaner, Lynch e Smith, 2000; Gil-Lacruz, e Gil-Lacruz, 2006). Cook e Moore (2001) e Campbell (2000) sosteñen que os individuos nunha 
comunidade se integran na medida en que o capital social é valorado en base ás prácticas propias tales como costumes e hábitos de convivencia, e, como consecuencia, o capital social constitúese sobre a base dos estudos do capital humano (Durkin, 2000; Lee e Croninger, 2001; Sutherland et al., 2001; Veenstra, 2001; Campbell e Jovchelovitch, 2000) e das potencialidades do desenvolvemento local (Castelleti e Canzanelli, 2005; Vázquez Barquero, 2009; Alburquerque, 2004). Baixo estes antecedentes, o presente estudo formula tres hipóteses que se pretenden probar: cantas máis actividades desenvolven en rede (alianzas estratéxicas), maior será o fortalecemento das organizacións comunitarias; canto maior sexa a confianza nas organizacións comunitarias, a súa contribución ao desenvolvemento local será de maior importancia, e, canto maior sexa a confianza nas redes, as organizacións comunitarias serán máis sostibles. Todas elas son contrastadas dende o punto de vista teórico e empírico.

\section{MÉTODOS E MATERIAIS}

A investigación aplica a metodoloxía analítica entre as formulacións teóricas desenvolvidas no eido do capital social e as súas implicacións no capital social comunitario. A consulta da literatura e bibliografía especializada coadxuva na comprensión e identificación das dimensións e aspectos que contempla o estudo do capital social, cuxos traballos desenvolvidos apoiaron o establecemento dun modelo de concepción nas comunidades rurais. O traballo realízase na provincia Avaroa do departamento de Oruro, ubicada na parte andina de Bolivia, cuxa poboación adicada á agricultura e gandaría que habita na área rural chega a 3.664 familias, das cales se determina unha mostra de 347 familias; a enquisa realízase ao xefe de familia, o cal permite apreciar as concentracións de poboacións no sector rural e identifica a contorna e as súas organizacións, ademais das principais actividades que desenvolven nas súas comunidades. As dimensións abordadas e formuladas nas hipóteses, son identificadas e suxeitas a contrastación empírica e dende o punto de vista teórico.

\section{RESULTADOS}

En primeiro lugar, preséntanse algunhas características da mostra que contempla o presente estudo. É interesante aportar algunhas cuestións que describen á poboación rural que participou na enquisa, e que se observan na seguinte táboa.

En canto ao sexo, o 70,03\% da poboación que participou na enquisa (347 en total) corresponde a homes e un $29,97 \%$ a mulleres. O grupo de idade con maior representatividade é de 31 a 40 anos que corresponde a un 28,24\%, seguido por maiores de 51, co 27,09\%; moi preto a esta porcentaxe atópase o grupo de 41 a 50 anos, en termos relativos representa o 21,24\%; continúa o grupo de 21 a 30 anos co 16,43\%, e finalmente o grupo menos representado son os que teñen menos de 20 anos, co 6,34\%. É destacable que os beneficiarios maiores de 31 anos suman o 76,57\%. Con respecto á formación escolar da poboación, os que non teñen ningunha formación chega ao 48,70\% moi preto do $50 \%$, séguelle unha poboación que representa o 29,97\% cuxa formación corresponde ao ensino primario, seguido pola formación intermedia co 14,97\%, a formación a nivel secundario representa o 6,34\%, e, finalmente, a poboación que ten unha formación universitaria representa o $0,29 \%$. Tamén se observa, que o nivel de formación da poboación que participou na enquisa suma o 78,67\% entre a formación primaria e aqueles que non contan con formación educativa. 
Táboa 1. Características da poboación

\begin{tabular}{|c|c|c|c|}
\hline Variables & Respostas & Frecuencias & Porcentaxe \\
\hline \multirow{2}{*}{ Sexo } & Home & 243 & 70,03 \\
\hline & Muller & 104 & 29,97 \\
\hline \multirow{5}{*}{ Idade } & Menos de 20 & 22 & 6,34 \\
\hline & De 21 a 30 & 57 & 16,43 \\
\hline & De 31 a 40 & 98 & 28,24 \\
\hline & De 41 a 50 & 76 & 21,24 \\
\hline & Máis de 51 & 94 & 27,09 \\
\hline \multirow{5}{*}{$\begin{array}{l}\text { Nivel de } \\
\text { estudos }\end{array}$} & Nivel primario & 104 & 29,97 \\
\hline & Nivel intermedio & 51 & 14,97 \\
\hline & Nivel secundario & 22 & 6,34 \\
\hline & Nivel Universitario & 1 & 0,29 \\
\hline & Sen formación & 169 & 48,70 \\
\hline
\end{tabular}

Fonte: Elaboración propia

Segundo a enquisa, o 85,87\% da poboación sinala que existen varias organizacións locais comunitarias, institucións que lideran e exercen autoridade para a posta en marcha de normativas, regulamentos e plans na comunidade, as mesmas son establecidas en base ao sistema organizativo e cultural das comunidades rurais, cuxa práctica provén dun sistema tradicional e baseado en costumes e saberes comunitarios propios de cada territorio. $\mathrm{O}$ traballo en rede e unha coordinación sistemática en proxectos locais permiten establecelos como un capital importante das comunidades. Por outra banda, as comunidades son beneficiarias de proxectos que executan as Organizacións Non Gobernamentais, e preto do $70 \%$ da poboación está implicada en proxectos agrícolas, créditos, gandaría e dereitos humanos, que están relacionados cos actores locais e implicados en proxectos de desenvolvemento e sociais, aspecto relevante da poboación na participación dos procesos de desenvolvemento local. Na táboa 2 obsérvase o comportamento das hipóteses formuladas para a investigación.

As hipóteses formuladas na táboa 2 , foron obxecto de análise a través da ji cadrada, a súa correlación e o $\mathrm{R}^{2}$, estatísticas que axudan na aceptación ou rexeitamento dunha hipótese (Monge e Pérez, 2009; Cobo, 2007; De la Horra, 2003; Castro, 2000; Montanero, 2008). Como se observa, o axuste do modelo aos datos é aceptable, xa que os indicadores se atopan entre os valores recomendados. Nas correlacións entre os distintos construtos, estas amosan valores positivos e significativos; do mesmo xeito a ji cadrada asume un 
valor de $\mathrm{p}<0,05$, polo que se rexeita a hipótese nula de independencia e, como consecuencia, as variables estudadas en cada hipótese, corresponden a unha función de dependencia unha da outra. Porén, na última hipótese, o $\mathrm{R}^{2}$ ten un grao de significación moi pequeno.

Táboa 2. Resultados da estimación do modelo global

\begin{tabular}{lccc}
\hline \multicolumn{1}{c}{ Relación no modelo } & J cadrada & Correl & $\mathbf{R}^{\mathbf{2}}$ \\
\hline $\begin{array}{l}\text { H1. Cantas máis actividades desenvolven en rede } \\
\text { (alianzas estratéxicas), maior será o fortalecemento das } \\
\text { organizacións comunitarias. }\end{array}$ & $\begin{array}{l}\mathrm{gl}=38,82 \\
\mathrm{p}=0,021\end{array}$ & 0,90 & 0,87 \\
\hline $\begin{array}{l}\text { H2. Canto maior sexa a confianza nas organizacións } \\
\text { comunitarias, a súa contribución no desenvolvemento } \\
\text { local é de maior importancia. }\end{array}$ & $\begin{array}{l}\mathrm{gl}=84,16 \\
\mathrm{p}=0,013\end{array}$ & 0,74 & 0,66 \\
\hline $\begin{array}{l}\text { H3. Canto maior sexa a confianza nas redes, as } \\
\text { organizacións comunitarias serán máis sostibles. }\end{array}$ & $\begin{array}{l}\mathrm{gl}=46,24 \\
\mathrm{p}=0,044\end{array}$ & 0,68 & 0,51 \\
\hline
\end{tabular}

Na primeira hipótese (H1), a relación entre as actividades que desenvolven en redes e o fortalecemento das organizacións comunitarias, non se atopa evidencia en contra para rexeitar a devandita afirmación. Aparece o valor de $\mathrm{p}=0,021$, unha correlación de 0,90 , e un $\mathrm{R} 2=0,87$, este último mide a proporción de variabilidade da variable dependente explicada pola variable independente. Nesa liña, investigadores como Yánez (1990), Carleen et al., (2002), Requena (2003) Villasante e Martín, (2006), Moreno (2007), Gutiérrez e Flores (2011) sinalan que as redes coadxuvan no crecemento e fortalecemento das organizacioóns locais. As actividades na área rural desenvólvense baixo a figura de asociatividade e cooperación, esta última entendida como a minka ou minga (palabra en quechua), baseada nunha relación de apoio mutuo e de xeración de confianza interna, polo que, no eido das comunidades rurais, o capital social está enraízado nos seus costumes e formas propias de traballo en redes sociais densas, e, como consecuencia, fortalecen ás organizacións comunitarias.

A segunda hipótese (H2), amosa un valor de $\mathrm{p}=0,013$, unha correlación 0,74 e un $\mathrm{R} 2=0,66$; estes datos confirman que a formulación, canto maior sexa a confianza nas organizacións comunitarias, a súa contribución ao desenvolvemento local é de maior importancia, valídase e está sustentada pola relación que existe entre as variables; de igual forma, existen autores que se achegan á formulación da confianza nas organizacións e estas teñen resultados na xeración de valores na xestión e a mellora nas súas intervencións locais (Loli, 1988; Guinalíu, 2003; Pena López e Sánchez, 2005; Frías et al., 2011; Mendieta e Araujo, 2012). A asociación de persoas, organizacións e comunidades cos mesmos intereses, e de semellantes condicións, sociais, económicas e culturais fortalece a confianza e empodera aos membros da mesma a procurar obxectivos comúns. Como consecuencia, a hipótese é validada tanto estatísticamente e dende o punto de vista teórico. 
Os resultados da terceira hipótese (H3), canto maior sexa a confianza nas redes, as organizacións comunitarias serán máis sostible, proporciona a seguinte información, $\mathrm{p}=0,044$, unha correlación de 0,68 e un $\mathrm{R} 2=0,51$. A proporción de variabilidade da variable dependente explicada pola variable independente é menor en relación ás outras dimensións, razón pola que se pode afirmar que existe unha feble relación e non altamente significativa. Esta formulación coincide con Luna e Velasco (2005) e Aguerre (2011) que manifestan que a confianza nas redes están dadas na medida en que os individuos se identifiquen con elas e podería determinar a continuidade ou o divorcio daquelas organizacións que a conforman.

\section{DISCUSIÓN}

A investigación identifica tres dimensións que forman parte do capital social, a rede, organizacións comunitarias e confianza, que actúan e se relacionan entre elas, a partir destas dimensións, as comunidades rurais impulsan o fortalecemento dos procesos e a procura do desenvolvemento local. A estas variables, a teoría defíneas como dimensións do capital social, dado que son variables cualitativas e propias de cada territorio, dependerá da cultura e identidade a súa forma de organización, a participación en redes e a confianza que teñan na contorna e cambios que produce o desenvolvemento local. As redes sociais densas ou fortes son as que xeran confianza entre os membros das mesmas, ao existir esta confianza, desenvólvense eficientemente os termos de intercambio, e polo tanto, redúcense os custos de transacción; á súa vez, esta interrelación entre persoas, institucións e comunidades crean normas e regras de xogo que definen unha estrutura local de convivencia; polo tanto, o capital social é un indicador importante en termos económicos e de estrutura legal e social. Porén, tamén é importante mencionar que nos procesos de desenvolvemento interveñen outras forzas e variables que converxen nese propósito.

Dende a visión teórica, o capital social incorpora varias variables de análise segundo o contexto no que se aplica e desenvolve o concepto (Schiff, 1992; Lesser, 2000; Winter, 2000; Stone, 2000; Kliksberg, 2000; Roche, 2004; Van Deth, 2008; Martínez, 2010; Sánchez, 2013; Haz, 2015), estas identifícanse e son relevantes na medida en que esas prácticas son axeitadas ou forman parte da cultura ou sistemas de vida dos individuos ou grupos (Levy e Varnagy, 2005; Román et al., 2013; Christoforou, 2013; Villalonga, 2015; Jha e Cox, 2015). Así, as variables ou dimensións analizadas no contexto das comunidades rurais, xogan un papel importante na configuración do capital social, dimensións propias nos seus sistemas organizativos e de relacionamiento comunitario. Habería que engadir á análise outras cuestións que poderían ser obxecto de estudo, como ser a lealdade e a cultura nativa, costumes e saberes ancestrais, de tal forma, coñecer en que medida estes compoñentes inflúen no concepto do capital social no contexto das comunidades rurais e a súa relación co desenvolvemento local.

\section{CONCLUSIÓNS}

A revisión teórica establece que as dimensións analizadas na investigación se relacionan co capital social, e as prácticas en comunidades rurais están presentes como unha forma de xestión e intervención das actividades locais; estas accións configuran un escenario de coordinación e fortalecemento das súas organizacións en base a un dos principios de convivencia comunitaria, como é a confianza nas súas organizacións como mecanismo de desenvolvemento sostido dos territorios. 
A participación en redes como un sistema de traballo intercomunitario, na que participan comunidades veciñas nos proxectos comunitarios e de cooperación internacional, outórgalles un valor social importante na consecución e cumprimento dos obxectivos locais; así tamén, o papel que xogan as organizacións comunitarias no desenvolvemento local se visibiliza na confianza que adquire a poboación nelas, e, como consecuencia, constitúese como un elemento do capital social no eido rural.

Finalmente, as dimensións das redes, organizacións comunitarias e a confianza relaciónanse estatísticamente e contribúen na explicación do capital social nas comunidades rurais; malia que a última variable asociada á sostibilidade das organizacións comunitarias, non é moi representativa; porén, obsérvase a relación entre elas. Así, a investigación conclúe afirmando que estas dimensións contemplan e contribúen ao capital social comunitario, prácticas ancestrais que estiveron presentes nas súas formas e sistemas de xestión e convivencia.

\section{REFERENCIAS BIBLIOGRÁFICAS}

AGUERRE, C. (2011): "Comunicación, stakeholders y las redes de confianza en las organizaciones", Dixit, (15), pp. 34-38.

ALBURQUERQUE, F. (2004): “Desarrollo económico local y descentralización en América Latina", Revista de la Cepal, (82), pp. 157-171.

BALLESTER, L; PASCUAL, B. Y VECINA, C. (2014): "Redes sociales, políticas públicas y capital social", Aposta: Revista de ciencias sociales, (61), pp. 28-43.

BOURDIEU, P. (1988): Las formas del capital; capital económico, capital cultural y capital social, En: Poder Derecho y Clases Sociales: 131-165. №5, Colección Clásicos Contemporáneos. Ed. Centro de Investigaciones Sociológicas (CIS) (Madrid).

CALLOWAY, M. Y NADLICKI, T.M. (2000): Research and learning: using theories of social capital to guide health policy and evaluation. Association Paper. American Sociological Association. USA.

CAMPBELL, C. (2000): Social capital in health: Contextualizing health promotion within local community networks. En S. Baron, J.Field y T. Schuller (Eds.). Social capital: Critical Perspectives. Oxford. Oxford University Press.

CAMPBELL, C. Y JOVCHELOVITCH, S. (2000): "Health, Community and Development: Towards a Social Psychology of Participation", Journal of Community and Applied Social Psychology, (10), pp. 225-270.

CARLEEN, R.; COLLER, X. Y ANDREWS, S. (2002): "Redes, cultura e identidad en las organizaciones", Reis: Revista española de investigaciones sociológicas, (97), pp. 31-56.

CARPIO, J. (2001): “Desarrollo local en los espacios rurales", Polis, Revista de la Universidad Bolivariana, 1 (2), pp. 45-56. 
CASTELLETTI, O. Y CANZANELLI, G. (2005): "Estrategias e instrumentos para el desarrollo local en la era de la globalización". Revista Ópera, abril, (5), pp. 78-88.

CHRISTOFOROU, A. (2013): "On the identity of social capital and the social capital of identity", Cambridge journal of economics, (4), pp. 719-736.

COBO, E. (2007): Bioestadística para no estadísticos: Bases para interpretar artículos científicos, Elsevier Masson.

COOK, P. Y MOORE, M. (2001): Environment and persistence in youthful drinking patterns. En Gruber, J. (eds). Risky behaviour among youths: an economic analysis. Chicago: Chicago Press.

DE LA HORRA, J. (2003): Estadística Aplicada, Díaz de Santos. España.

CASTRO, J. Y GALINDO, M.P. (2000): Estadística Multivariante: Análisis de correlaciones, Amaru Ediciones, Madrid.

DELHEY, J. Y NEWTON, K. (2002): Who Trusts? The Origins of Social Trust in Seven Nations, Berlin, Social Science Research Center Berlin.

DODD, M.; BRUMMETTE, J. Y HAZLETON, V. (2015): "A social capital approach: An examination of Putnam's civic engagement and public relations roles", Public Relations Review, 41 (4), pp. 472-479.

DURKIN, J. (2000): Measuring social capitaland its economic impact. Working Paper, University of Chicago.

FALLETO, E. Y MARTNER, G. (1990): Repensar el Futuro, Estilos de Desarrollo. Compilación de Ensayos. Editorial Nueva Sociedad.

FRÍAS, R; FELLINGER, E. Y CLARKE, D. (2011): "El rol de la confianza en las organizaciones", Capital humano: revista para la integración y desarrollo de los recursos humanos, (258), pp. 98-105.

FORNI, P.; NARDONE, M. Y CASTRONUOVO, L. (2013): "Capital social y organización comunitaria: la urbanización del Barrio Almafuerte, Pilquen" - Sección Ciencias Sociales, 16 (2), pp. 1- 14.

FORNI, P.; CASTRONUOVO, L. Y NARDONE, M. (2012): "Las organizaciones en red y la generación de capital social. Implicancias para el desarrollo comunitario", Miríada: Investigación en Ciencias Sociales, 4 (8), pp. 79-106.

FUKUYAMA, F. (2001): "Social capital, civil society, and development", Third World Quarterly, 22 (5), pp. 47-61.

GIL-LACRUZ, M. Y GIL-LACRUZ, A. I. (2006): "Del capital humano al capital social: estrategias de prevención del consumo de alcohol", Revista Originales, (31), pp. 79-92. 
GORDON, S. (2005): "Confianza, capital social y desempeño de organizaciones", Revista Mexicana de Ciencias Politicas y Sociales, 47 (193), pp. 41-55.

GUTIÉRREZ GONZÁLEZ, L. Y FLORES, M. (2011): “Un concepto sobre las redes de conocimiento entre organizaciones", Revista de ciencias sociales, 17 (3), pp. 473-485.

GUINALÍU, A. (2003): Confianza en el e-government: factores antecedentes de la confianza del ciudadano en la prestación de servicios públicos online, Aplicaciones del marketing en la administración pública y organizaciones no lucrativas, Madrid.

GUIA JULVE, J. (1999): “Capital social, redes relacionales y creación de conocimientos en las organizaciones", Revista de economía y empresa, 13 (37), pp. 55-82.

GUTIÉRREZ, S. (2016): "Capital Social, cultura organizacional, cultura innovadora y su incidencia en las organizaciones productivas rurales colaborativas", Revista Economía y Sociedad, (34), pp. 119-136.

HAZ, F. (2015): El capital social y la Universidad: Estudio sobre la influencia de la Universidad en la construcción del capital social en los jóvenes. Tesis Doctoral, Universidad de la Coruña, España.

IBÁÑEZ, E.; RAMIREZ DE HARO, G. Y CUERDO, M. (2004): El capital social y el estudio del desarrollo económico, Universidad Rey Juan Carlos, Madrid.

JHA, A. Y COX, J. (2015): "Corporate social responsibility and social capital", Journal of banking and finance, 60 (11), pp. 252-270.

KINGSTON, C. Y CABALLERO, G. (2005): "Capital social e instituciones en el proceso de cambio económico", Ekonomiaz: Revista vasca de economía, №. 59, 2005 (Ejemplar dedicado a: Capital social: Innovación organizativa y desarrollo económico), pp. 70-91.

KLIKSBERG, B. (2000): Capital social y cultura: claves olvidadas del desarrollo. Ed. Banco Interamericano de Desarrollo, Departamento de Integración y Programas Regionales, (Washington) Estados Unidos.

LEE, V. Y CRONINGER, R. (2001): "The elements of social capital in the context of six high schools", Journal of Socio-Economics, 30: 165-167.

LESSER, E. (2000): Knowledge and Social Capital. Global Real. USA.

LEVI, M. (1996): "Social and Unsocial Capital: A Review Essay of Robert Putnam's Making Democracy Work”, Politics \& Society, № 24, en Zona Abierta, (94-95), pp. 105-119.

LEVY, S. Y VARNAGY, D. (2005): "Capital financiero y capital social: dos ingredientes del desarrollo", Líder: revista labor interdisciplinaria de desarrollo regional, (13), pp. 13-30.

LIN, N. (2001): Social Capital: A Theory of Social Structure and Action. Ed. Cambridge University Press (Cambridge) UK. 
LOL, A. (1988). La confianza interpersonal en las cooperativas del Perú, Psicología, trabajo, organización y nuevas formas de empleo: 1er Congreso Iberoamericano y $3^{\circ}$ Nacional de Psicología del Trabajo y de las Organizaciones, Madrid.

LUNA, M. Y VELASCO, J. L. (2005): “Confianza y desempeño en las redes sociales”, Revista mexicana de sociología, 67 (1), pp. 127-162.

MARGARIDA, I. Y REGO, P. (2003): “Redes y desarrollo local: la importancia del capital social y de la innovación", Boletín de la Asociación de Geógrafos Españoles, (36), pp. 117127.

MARTÍ OLIVÉ, J. Y LOZARES COLINA, C. (2008): "Redes organizativas locales y capital social: enfoques complementarios desde el análisis de redes sociales", Portularia: Revista de Trabajo Social, 8 (1), 23-39.

MARTINEZ-CÁRDENAS, R.; AYALA-GAYTÁN, E. Y AGUAYO-TELLEZ, S. (2015): “Confianza y capital social: evidencia para México", Economía, Sociedad y Territorio, 15 (47), pp. 35-59.

MARTÍNEZ, J. (2010): Capital Social y desarrollo económico en Puebla, México, Desarrollo local y cooperación internacional / coord. por Eugenio Sánchez Alcázar, pp. 57-88.

MATUS, C. (1986): Política, Planificación y Gobierno, Instituto Latinoamericano y del Caribe de Planificación Económica y Social (ILPES), Fundación Altadir.

MAVARES, A.; DÍAZ, M.; COLINA, F.; LOMBARDI, D. Y PRIETO, J. (1999): “El capital humano, el capital social y su importancia para el desarrollo económico", Revista de ciencias sociales, 5 (2), pp. 129-136.

MENDIETA, A. Y ARAUJO, L. (2012): La confianza en las Organizaciones No Gubernamentales, Actas IV Congreso Internacional Latina de Comunicación Social: Comunicación, control y resistencias / coord. por Concha Mateos Martín. La Laguna.

MONGE, J.F. Y PÉREZ, A. (2009): "Estadística No Paramétrica: prueba de chi cuadrado", Cuadernos de trabajo, UOC, Págs. 1-20.

MONTANERO, J. (2008): Análisis Multivariante, Colección de manuales UEX-59. Universidad de Extremadura.

MORENO, A. (2007): Las organizaciones en red y sus nuevas identidades, Tesis doctoral, UNED, España.

MOYANO, E. (2002): "Capital social y desarrollo en zonas rurales: Un análisis de los programas Leader II y Proder en Andalucía", Revista internacional de sociología, (33), pp. 67-96.

MUNTANER, C., LYNCH, J. Y SMITH, G.D. (2000): "Social capital and the third way in public health", Critical Public Health, (10), pp. 107-124. 
PENA LÓPEZ, J. A. Y SÁNCHEZ SANTOS, J. M. (2005): “Actividad asociativa, confianza y generación de capital social: evidencia empírica", Ekonomiaz: Revista vasca de economía, (59), pp. 136-159.

PENA, J. A. Y SÁNCHEZ, J. M. (2013): El capital social individual: lo micro y lo macro en las relaciones sociales. En: Veira, J. L. (coord.)Capital social y desigualdad en España. Ed. Netbiblo (A Coruña).

PNUD / BID (1998). El Capital Social. Hacia la Construcción del Índice de Desarrollo Sociedad Civil de Argentina, Edilab Editora, Buenos Aires.

POSNER, D. Y BOIX, C. (2000): "Capital social y democracia”, Revista española de ciencia política, (2), pp. 159-186.

PORTES, A. (2000): "The Two Meanings of Social Capital”, Sociological Forum, 15 (1), pp. $1-12$.

PUTNAM, R. (2001): "Social Capital: Measurement and Consequences Isuma: Canadian", Journal of Policy Research. (2), pp. 41-51.

PUTNAM, R. (2003): El declive del capital social: un estudio internacional sobre las sociedades y el sentido comunitario, Barcelona: Galaxia Gutenberg.

REQUENA SANTOS, F. (2003): Análisis de redes sociales. Orígenes, teorías y aplicaciones, CIS - Colección Monografías, Madrid.

ROCHE, M. (2004): "Social Policy and Social Capital: A Clear Case of Putting Merit before Method?", Social Policy and Society, 3 (2), pp. 97-111.

ROMÁN, R. E., GÓMEZ, A. Y SMDA, A. (2013): "El capital social organizacional de la pequena empresa innovadora. Un ensayo de medición en las ciudades de Cali y Medellín". Estudios Gerenciales, 29 (128), pp. 356-367.

SÁNCHEZ, V. (2013): "El capital social como instrumento del análisis económico", Cuadernos de relaciones laborales, 31 (2), pp. 473-493.

SAZ GIL. M. I. (2006): El capital social en las organizaciones no lucrativas. Implicaciones en la gestión: Una aproximación a través del estudio de casos, Tesis doctoral, Universitat de València, España.

Schiff, M. (1992): "Social Capital, Labor Mobility, and Welfare", Rationality and Society, (4), pp. 157-175.

SCULL, S. (2001): Social Capital; A briefing paper for Module Development. West Morton Public Health, Unit Queensland Health, Australia.

Stone, W. (2000): "Social Capital, social cohesion and social security", Journal of Economic Literature, (40), pp. 175-154. 
SUTHERLAND, I. Y SHEPHERD, J. (2001): The prevalence of alcohol, cigarette and illicit drug use in a stratified sample of English adolescents". Revista Addiction, (96), pp. 637640.

SUDARSKY, J. (2004): "Las llaves del desarrollo económico y social", Cuadernos Unimetanos, (1), pp. 4-13.

THIEBAULT, J. L. (2003): "Les travaux de Robert D. Putnam sur la confiance, le capital social, l'engagement civique et la politique comparée", Revue internationale de politique comparée, 10 (3), pp. 341-355.

URTEAGA OLANO, E. (2013): "La teoría del capital social de Robert Putnam: Originalidad y carencias”, Reflexión política, 15 (29), pp. 3-17.

VAN DETH, J. W. (2008): Measuring Social Capital, en Castiglione, D.; Van Deth, J. W. and Wolleb, G. (Ed.) The handbook of social capital. Oxford University Press (Oxford) UK.

VÁZQUEZ-BARQUERO, A. (2009): Desarrollo local, una estrategia para tiempos de crisis. Universidad Autónoma de Madrid. Trabajo presentado en el Seminario Internacional de la Red DETE-ALC, Rafaela, Argentina.

VEENSTRA, G. (2001): Social Capital and Health. Printemps, Spring, Chicago.

VILLASANTE, T. Y MARTÍN, P. (2006): “Redes y conjuntos de acción: para aplicaciones estratégicas en los tiempos de la complejidad social”, EDES-Revista hispana para el análisis de redes sociales, 11 (2), pp. 34-51.

VILLALONGA, E. Y KAWACHI, I. (2015): "The measurement of social capital”, Gaceta sanitaria: Organo oficial de la Sociedad Española de Salud Pública y Administración Sanitaria, 29 (1), pp. 62-64.

WINTER, I. (2000): Family Life and Social Capital: towards a theorised understanding, Working Paper, Australian Institute of Family Studies, (Melbourne) Australia.

YÁNEZ, M. (1990): "Redes y organizaciones, Chasqui", Revista Latinoamericana de Comunicación, (34), pp. 25-27.

Revista Galega de Economía: http:/ / www.usc.es/ econo/ RGE/ benvidag.htm 